\title{
Subtrochanteric shortening osteotomy during cementless total hip arthroplasty in young patients with severe developmental dysplasia of the hip
}

Duan Wang ${ }^{1 \dagger}$, De-Hua Li ${ }^{2,3+}$, Qi Li ${ }^{1 \dagger}$, Hao-Yang Wang ${ }^{1 \dagger}$, Ze-Yu Luo ${ }^{1}$, Yang Yang ${ }^{4}$, Fu-Xing Pei ${ }^{1}$ and Zong-Ke Zhou ${ }^{1 *}$ (D)

\begin{abstract}
Background: This retrospective study was designed to determine complications, functional and radiographic results of transverse subtrochanteric osteotomy during cementless, modular total hip arthroplasty (THA) in a series of active patients younger than 45 years with Crowe Type-III or IV developmental dysplasia of the hip (DDH).

Methods: We followed 49 patients (56 hips) with DDH who were treated with cementless THA, where the acetabular cup was positioned in the anatomic hip center and where a simultaneous transverse femoral osteotomy was performed. Complication rate evaluation and clinical outcomes were measured by validated clinical scores and radiographic evaluation were performed at a mean follow up of 10 years (range, 4.8-14.3 years).

Results: The mean limb-length discrepancy was reduced from $4.2 \mathrm{~cm}$ to $1.1 \mathrm{~cm}(P<0.01)$. The mean Harris hip score (HSS) significantly improved from 40.6 points to 87.4 points $(P<0.01)$. Similarly, severity of low back pain, modified MAP, HOOS, and SF-12 also showed significant improvement $(P<0.01)$. There were 3 cases of postoperative dislocation, 3 cases of transient nerve palsy, 2 cases of nonunion, and 4 cases of intraoperative fracture. At 10 years follow-up, the estimated survival rate with any component revision as end points was $92 \%$.
\end{abstract}

Conclusion: The cementless THA combined with transverse subtrochanteric osteotomy is a reliable technique with restoration of a more normal limb, satisfactory clinical outcomes, and mid-term survival of components.

\section{Background}

Characterized by anatomical abnormalities, biomechanical alterations, femoral deformities and severe soft tissue contractures, severe developmental dysplasia of the hip (DDH) increases the complexity of performing total hip arthroplasty (THA) [1, 2]. In most patients with Crowe Type-III or IV DDH, the true acetabulum is the best position for supporting cup due to sufficient bone stock and good biomechanics [3]. Therefore, placement of the acetabular cup in the true acetabulum has proved to obtain durable clinical and favorable biomechanical

\footnotetext{
* Correspondence: zongke@126.com

${ }^{\dagger}$ Equal contributors

'Department of Orthopedics, West China Hospital/West China School of Medicine, Sichuan University, 37\# Wuhou Guoxue road, Chengdu 610041, People's Republic of China

Full list of author information is available at the end of the article
}

results of THA in such patients [4-6]. However, the limb may be lengthened by more than $4 \mathrm{~cm}$ during restoration of the anatomical hip rotation center, which resulted in overstretching of neurovascular structures and difficulty in reducing the hip. In addition, overstretching of peri-articular structures, including muscles and tendons, may lead to the dysfunction of the abductor muscles, hip stiffness, and early loosening of the components [7].

To address these concerns during THA for severe dysplasia, femoral shortening is sometimes necessary and an effective in most cases to correct femoral malrotation, facilitate the reduction, and equalize limb lengths without tension forces and increased risk of neurological traction injury $[5,7,8]$. Shortening osteotomy at different anatomic levels, including greater trochanter, 
subtrochanteric region,, and distal femur, has been described previously. Subtrochanteric osteotomies can be performed with various techniques, such as transverse, oblique, Z-shaped, and chevron-shaped, each of which has their own advantages and disadvantages [2, 9-11]. The main disadvantage of the osteotomy is the risk of fracture and nonunion. The transverse type of bone cutting is a simpler and effective technique with similar rotational stability compared with other types of osteotomy in young patients [10]. Compared with a non-modular standard stem, a modular stem may be desirable to provide good fit and fill in the femoral canal and to obtain torsional stability at the osteotomy site [12]. In our center, we have performed cementless THA combined with transverse subtrochanteric osteotomy with use of modular stem in most patients with severe dysplasia [13].

Several prior reports have described short- to mid-term results of cementless THA combined femoral osteotomy shortening using modular stem in limited number of patients with severe dysplasia. However, these reports have included mixed groups of cemented and cementless femoral and acetabular components, different types of femoral osteotomy techniques, or even no femoral osteotomy in patients with large age span [9, 11, 14-16]. Moreover, many patients with severe DDH have complained not only of hip pain but also of low back pain and needed intensive treatment for such pain [17]. Lumbar spine and hip arthritis often coexist. Some studies demonstrated that THA could relieve the low back pain in patients with hip arthritis [18]. Nevertheless, no previous studies evaluated the low back pain after THA combined with femoral shorting osteotomy in active young patients who present severe hip dysplasia.

The purpose of the present study was to evaluated (1) mid-term clinical outcomes, (2) radiographic evaluation, (3) complications, and (4) mid-term survival of components in a group of active young patients with Crowe type-III and IV DDH who underwent cementless THA with transverse simultaneous subtrochanteric shortening osteotomy. We hypothesized that the cementless THA combined with transverse subtrochanteric shortening osteotomy is a reliable method in restoration of a more normal limb and satisfactory clinical outcomes, with mid-term survival of components for young patients with Crowe type-III and IV DDH.

\section{Methods}

\section{Ethics statement}

The study protocol was approved by the local institutional review board of West China Hospital, Sichuan University. Informed consent (including patients' details, images, or videos) was obtained from all participants. All experiments were performed in accordance with relevant guidelines and regulations.

\section{Patients}

We retrospectively reviewed 53 consecutive patients $(62$ hips) in whom cementless THA combined with transverse subtrochanteric shortening osteotomies was performed for Type-III or IV DDH according to Crowe classification from 2002 to 2012 in joint registry. One patient (1 hip) did not attend due to health issues unrelated to the THA, and one patient (2 hips) did not attend owing to no longer being interested. Two patients (3 hips) were lost to follow up after surgery and could not be contacted by phone or email at least 5 attempts. In the remaining 49 patients ( 56 hips), the hip dislocation was presented unilaterally in 42 patients and bilaterally in 7 patients. In bilateral high dislocations, 5 patients had Crowe type-IV, and 2 patients had Crowe type-IV dislocation of one hip and Type-III of the other. Therefore, 8 hips (14.3\%) were Crowe Type III, while the other 48 hips $(85.7 \%)$ were Crowe Type IV.

Severe pain unresponsive to non-operative management, pelvic obliquity, and functional impairment with limp in daily activities were the indication for surgery. There were 9 males and 40 females with mean age of 36.9 years (range, 19-45 years) at the time of index THA. Previous Schanz osteotomy was performed in two patients. Modular femoral stem (S-ROM', DePuy Orthopadics, Marsaw IN.) with porous-coated acetabular component (PINNACLE ${ }^{\circ}$, POROCOAT ${ }^{\circ}$, DePuy Orthopadics, Marsaw IN.) was identical in all hips. The mean postoperative follow-up period was 10.2 years (range, 4.8-14.3 years). The pre-, peri- and post-operative data and surgical data were prospectively collected onto a predefined data collection form for patients with DDH. Table 1 showed the demographics of our study group.

\section{Surgical planning and procedures}

All operations were planned with transparencies and performed with use of cementless femoral and acetabular components. An acetabular template was placed at anatomical hip center, and a femoral template was correspondingly interiorized. The amount of femoral shortening was calculated to improve leg length discrepancy and lengthen the leg by no more than 3$4 \mathrm{~cm}$ to avoid nerve injury. Three-dimensional computed tomography (CT) scans was utilized to evaluate the acetabular bone stock.

All patients were operated on using a posterolateral approach in the lateral decubitus position. After resection of the femoral head and total capsulectomy were conducted, the position of the true acetabulum was reached and exposed through removal of fibrous scar tissue and osteophyte covering the true acetabulum. Softtissue release was applied in all hips. Transverse and round ligament was good indicator for recognizing true acetabulum. If the true acetabulum was not reachable 
Table 1 Demographics and clinical results

\begin{tabular}{|c|c|c|c|c|c|}
\hline \multirow[t]{2}{*}{ Indicator } & \multicolumn{2}{|c|}{ Pre-op clinical features } & \multirow{2}{*}{$\begin{array}{l}\text { Overall } \\
\text { (Pre-op) }\end{array}$} & \multirow{2}{*}{$\begin{array}{l}\text { Overall } \\
\text { (Post-op) }\end{array}$} & \multirow[t]{2}{*}{$P$ Value } \\
\hline & Crowe Type-III & Crowe Type-IV & & & \\
\hline No. of hips & 8 & 48 & 56 & 56 & \\
\hline \multicolumn{6}{|l|}{ Gender (No. of pts) } \\
\hline Male & 0 & 9 & 9 & - & \\
\hline Female & 8 & 34 & 40 & - & \\
\hline \multicolumn{6}{|l|}{ Affected side (No. of hips) } \\
\hline Left & 3 & 21 & 24 & - & \\
\hline Right & 5 & 17 & 22 & - & \\
\hline Bilateral & 0 & 10 & 10 & - & \\
\hline \multicolumn{6}{|l|}{ Harris Hip Score } \\
\hline Mean in points (range) & $54.6(48-59)$ & $38.2(23-57)$ & $40.6(23-59)$ & $87.4(77-98)$ & $<0.01$ \\
\hline \multicolumn{6}{|l|}{ Rating (no. of hips) } \\
\hline Excellent (90-100 points) & 0 & 0 & 0 & 15 & \\
\hline Good (80-89 points) & 0 & 0 & 0 & 39 & \\
\hline Fair (70-79 points) & 0 & 0 & 0 & 2 & \\
\hline Poor $(<70)$ & 8 & 48 & 56 & 0 & \\
\hline \multicolumn{6}{|l|}{ PMA } \\
\hline Mean in points (range) & $7.6(6-9)$ & $6.5(4-9)$ & $6.6(4-12)$ & $16(14-18)$ & $<0.01$ \\
\hline Pain & $2.5(2-3)$ & $2.1(1-4)$ & $2.2(1-4)$ & $5.5(5-6)$ & $<0.01$ \\
\hline Motion & $2.4(2-3)$ & $2.3(1-3)$ & $2.3(1-3)$ & $5.2(5-6)$ & $<0.01$ \\
\hline Function & $2.6(2-3)$ & $2.0(1-3)$ & $2.1(1-3)$ & $5.3(4-6)$ & $<0.01$ \\
\hline
\end{tabular}

Rating (no. of hips)

\section{Excellent (18 points)}

Good (15-18 points)

Fair (12-15 points)

Poor (<12 points)

Limp (no. of Pts.)

$\begin{array}{ll}\text { Severe } & 3 \\ \text { Moderate } & 4 \\ \text { Slight } & 1 \\ \text { None } & 0\end{array}$

Limb-length discrepancy

Mean in $\mathrm{mm}$ (range)

$<1 \mathrm{~cm}$ (no. of hips)

$1-2 \mathrm{~cm}$ (no. of hips)

2-3 cm (no. of hips)

3-4 cm (no. of hips)

4-5 cm (no. of hips)

5-6 cm (no. of hips)

$>6 \mathrm{~cm}$ (no. of hips)

HOOS subscale scores

Symptoms
Pain
Activities of daily living

$10.8(8-12)$

$20.6(17-24)$

$34.1(32-37)$

$2.8(2.1-3.7)$
0
0
5
3
0
0
0

0
0
0
48

48

28

15

5

0

$4.4(2.3-6.5)$
0
0
4
13
18
10
3
$8.8(4-12)$
$15.9(6-23)$

$28.7(19-36)$
0

0

0

56

$\begin{array}{ll}31 & 0 \\ 19 & 3 \\ 6 & 11 \\ 0 & 42\end{array}$

$\begin{array}{lll}4.2(2.1-6.5) & 1.1(0.6-1.4) & \\ 0 & 24 & \\ 0 & 32 & \\ 9 & 0 & \\ 16 & 0 & \\ 18 & 0 & \\ 10 & 0 & \\ 3 & 0 & <0.01 \\ 9.1(4-12) & 16.6(14-19) & <0.01 \\ 16.6(6-24) & 36.5(34-40) & <0.01 \\ 29.4(19-37) & 60.9(57-67) & \end{array}$


Table 1 Demographics and clinical results (Continued)

\begin{tabular}{|c|c|c|c|c|c|}
\hline \multirow[t]{2}{*}{ Indicator } & \multicolumn{2}{|c|}{ Pre-op clinical features } & \multirow{2}{*}{$\begin{array}{l}\text { Overall } \\
\text { (Pre-op) }\end{array}$} & \multirow{2}{*}{$\begin{array}{l}\text { Overall } \\
\text { (Post-op) }\end{array}$} & \multirow[t]{2}{*}{$P$ Value $^{a}$} \\
\hline & Crowe Type-III & Crowe Type-IV & & & \\
\hline Sports and recreation & $7.4(5-8)$ & $5.5(3-8)$ & $5.8(3-8)$ & $12.8(10-16)$ & $<0.01$ \\
\hline Quality of life & $6.9(5-8)$ & $5(3-8)$ & $5.3(3-8)$ & $13.7(12-16)$ & $<0.01$ \\
\hline \multicolumn{6}{|c|}{ Trendelenburg sign (no. of hips) } \\
\hline Yes & 6 & 47 & 53 & 1 & \\
\hline No & 2 & 1 & 3 & 55 & \\
\hline \multicolumn{6}{|l|}{ SF-12 } \\
\hline PCS & $15.5(12-17)$ & $10.9(6-16)$ & $11.6(6-17)$ & $22.7(19-25)$ & $<0.01$ \\
\hline MCS & $18.3(15-20)$ & $13.8(9-19)$ & $14.4(9-20)$ & $25.6(22-29)$ & $<0.01$ \\
\hline
\end{tabular}

Values are expressed as mean with range

MAP Merle d'Aubigne and Postel, PCS Physical component summary, MCS Mental component summary, ROM range of motion, HOOS Hip dysfunction and Osteoarthritis Outcome Score

${ }^{\text {a }} P<0.05$ is significant

due to the obstruction of the proximal part of the femur, a transverse femoral osteotomy approximately 1-2 cm distal to the lesser trochanter was performed. If there was proximal femoral canal deformity, an osteotomy was conducted at the level of the deformity. Elevation or split of a short section of the vastus lateralis was performed to approach the subtrochanteric area. Next, the proximal femoral fragment was translated anteriorly to reach the true acetabulum after the performance of transverse osteotomy. The acetabulum was widened and deepened with use of hemispherical reamers at a designated angle of antevertion and abduction to obtain bleeding cancellous, which indicated an interference fit between the anterior and posterior columns (Fig. 1a). As for insufficient coverage or severe acetabular bone defects, augmentation of the acetabulum was conducted by a structural autograft or titanium alloy (Ti-alloy) mesh combined with bulk bone grafting from the resected femoral head. Femoral head structural bone autografts fixed with screws were used in thirty-eight hips to improve coverage of the cup due to insufficient coverage, whereas Ti-alloy mesh and impaction bone grafting were used in nine hips owing to severe acetabular bone defects according to the three-dimensional CT. Implantation of the acetabular cup was conducted using press-fit technique (Fig. 1b). The median diameter of the acetabular cup was $48 \mathrm{~mm}$ (range, 44 to $52 \mathrm{~mm}$ ). The $28-\mathrm{mm}$ femoral head was used in twenty-six hips, 32-mm head in twenty-two hips, and $36-\mathrm{mm}$ head in eight hips. Ceramic-on-ceramic wear bearing was applied in fortyeight hips, and metal-on-poly in eight hips.

After the acetabular component was inserted, attention was then directed to the more distal femur. A second transverse subtrochanteric osteotomy was conducted to shorten the femur by the planned amount. If proximal femoral canal deformity existed, correction of rotational deformity of the proximal femur was conducted before axial reaming through subtrochanteric osteotomy. Four cases (4 hips) with such femoral canal deformity were corrected in our series. The straight axial intramedullary reaming process was conducted in the distal femur, and the proximal part of the femur was then prepared for the cementless implant (Fig.1c-d). Then, sequential rasping was performed until the appropriate femoral component size was achieved. The proximal and distal bone fragments were then aligned, and they were properly rotated on the trial component. If a trial reduction of the hip could not be achieved following the first cut of the osteotomy, the additional osteotomy was gradually performed in sequence also with a transverse design to archive satisfactory hip reduction. The average amount of femoral shorting was $2.7 \mathrm{~cm}$ (range, 1.9-4.5 cm). After the proximal sleeve was implanted, the straight stem was then inserted into the femur across the osteotomy site when the rotational alignment of the 2 fragments was adjusted to allow approximately $15^{\circ}-20^{\circ}$ of anteversion of the femoral component (Fig. 1e). If necessary, prophylactic cable fixation could be used to prevent femoral fracture. After the insertion of femoral component, the gap at the osteotomy site was grafted with autogenous morselized bone and stabilized by cable fixation to enhance biologic healing in forty-six cases (Fig. 1f). In most cases, cortical autograft struts cut from the resected femoral bone fragment was applied for additional stabilization of the osteotomy site. After the insertion of femoral and acetabular components, $\mathrm{C}$-arm X ray was used in most patients to ensure the position of the cup and stem (Fig. 1g).

\section{Postoperative rehabilitation}

All patients were encouraged to conduct isometric exercises and active motion on a bed immediately after surgery. The patients walked with partial weight bearing under the protection of crutches for approximately 2 weeks. Then, gradually progressive full weight bearing 


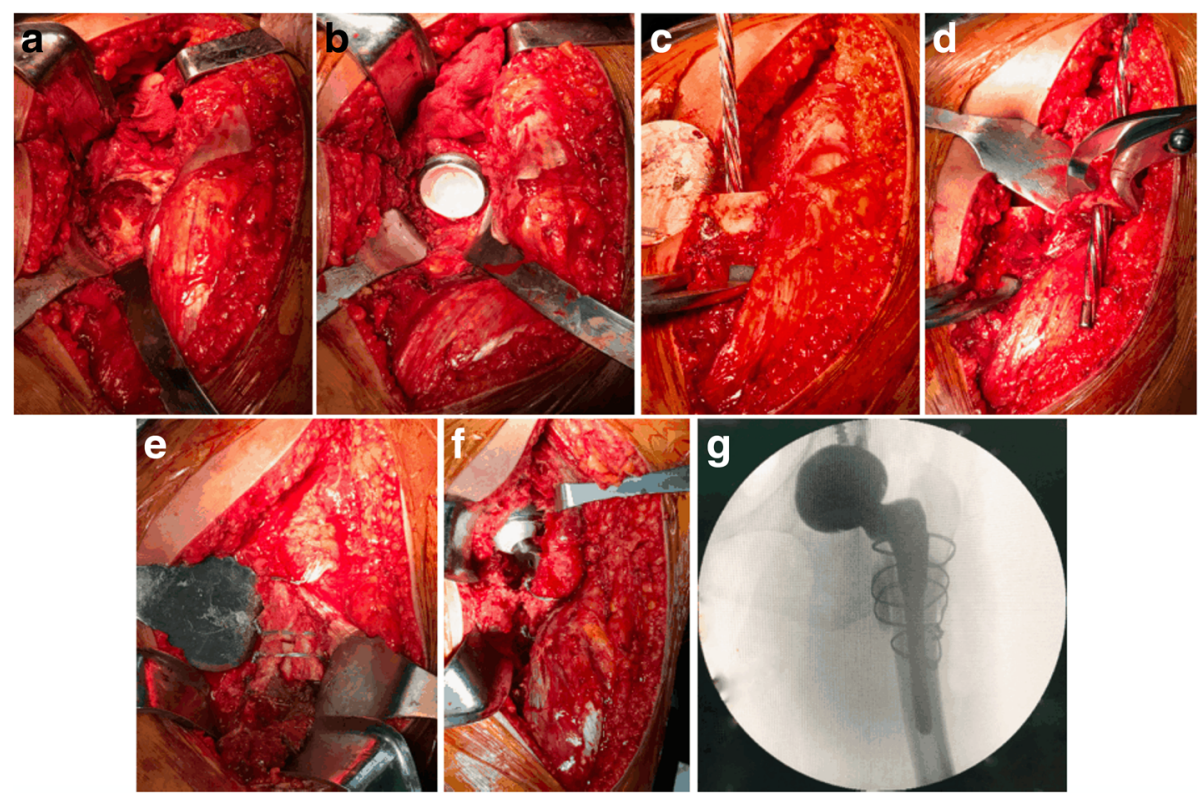

Fig. 1 Intraoperative photographs of THA in a thirty-one-year-old woman with Crowe type-IV DDH. (a) Acetabulum was widened and deepened to obtain bleeding cancellous and femoral head autograft was applied. (b) The insertion of acetabular cup. (c) The straight axial intramedullary reaming process was conducted in the distal femur. (d) The proximal part of the femur was then prepared for the cementless implant. (e-f) The straight stem was then inserted into the femur across the osteotomy site, at which the gap was grafted with autogenous morselized bone and stabilized by cable fixation. (g) C-arm $X$ ray was used to ensure the position of the cup and stem

was allowed 4-6 weeks depending on stability of the femoral stem and positively osseous healing at osteotomy site. The mean hospital stay was 7.2 days (range, 6-15 days).

\section{Clinical analysis}

Clinical evaluation was conducted at 3 weeks, 6 weeks, 12 weeks, and 6 months after the surgery and annually thereafter until the last follow-up. A informative, concise follow-up data correction form was designed by the senior author (Z.K.Z.) for the patients with DDH [13]. The form included Hip dysfunction and Osteoarthritis Outcome Score (HOOS) (covering pain, symptoms, daily living, Sports, and quality of life), which is a validated and highly reproducible tool for evaluation of patient's opinion about their hips, assessment of symptoms and functional limitations related to DDH, and description of their hip disability [19]. In addition, 12-item shortform health survey questionnaire (SF-12) is more sensitive than HOOS regarding the hip-related quality of life in younger active patients [13]. We decided to use HOOS and SF-12 as accurate measure of life quality and hip disability. In our center, the Harris Hip Score (HHS) and modified Merle d'Aubigne and Postel (MAP) hip score (including pain, motion, and gait function) are collected routinely to evaluate the function of the hip $[13,20,21]$. In addition to the validated patient-reported outcomes, all pre- and post-operative evaluations regarding the severity of limp and Trendelenburg test status were also achieved. The limb-length discrepancy (LLD) was also recorded in pre- and post-operative periods, which was measured from the anterior superior iliac spine to the medial malleolus suggesting the length discrepancy of lower extremities. At each visit, a specific questionnaire about the presence of low back pain and details of such pain was completed. We also asked the back-pain location and evaluated the intensity of the pain with use of the visual analogue scale (VAS). All complications were reviewed.

\section{Radiographic analysis}

At each visit, all patients had standard anteroposterior and lateral radiographs of the hip, full-length view of the lower extremities. All radiographic measurements were adjusted for magnification. Radiographs were evaluated by authors who were not involved in the surgery. The amount of limb length was calculated by subtracting the amount of intraoperative femoral shortening from the amount of difference between the pre- and postoperative tip of the greater trochanter [22]. Bone union of the osteotomy site was evaluated on serial radiographs with the following radiographic criteria, including the presence of callus, cortical continuity, and no progressive gapping at the osteotomy site [23]. In addition, we also evaluated whether and when bone union occurred at the osteotomy site. Serial radiographs were also 
examined with respect to peri-prosthetic radiolucency, component migration, heterotopic ossification, osteolysis, and subsidence. Peri-prosthetic radiolucency was documented in acetabulum and femoral components according Delee and Charnley [24]. Heterotopic ossification was classified according to the method of Brooker et al. [25]. The seven zones around the femoral component on radiographs were described by Gruen et al. [26]. The mode of fixation of the femoral component was classified as bone ingrown, fibrous stable or unstable according to the system of Engh et al. [27]. Subsidence of femoral stem was also evaluated according to the method described by Engh et al. [27]. The loosening of acetabular cup was defined by the presence of progressive radiolucent lines $>2 \mathrm{~mm}$ around the inserted cup, or migration, or a change in the position of the cup.

\section{Statistical analysis}

Two-sided paired Student $t$ test was used to analyze preoperative and postoperative continuous variables. Statistical significance was set at $P<0.05$. The $X^{2}$ test was performed to analyze categorical variables. These data are presented as mean values with ranges. Kaplan-Meier was used to conduct analysis of cumulative survival rate of acetabular and femoral components. The end points for survival were defined as revision for any reason (including radiographic signs of loosening at the latest follow-up). Statistical analysis was performed with the use of SPSS Statistics software version 19.0 (IBM, Armonk, NY).

\section{Results}

\section{Clinical outcomes}

The mean HHS improved significantly from 40.6 points (range, 23-59 points) preoperatively to 87.4 points (range, 77-98 points) by the final follow-up $(P<0.01)$. Similarly, the mean modified MAP hip score improved from 6.6 points (range, 4 to 12 points) preoperatively to 16 points (range, 14 to 18 points) at the time of the latest follow-up, and the difference revealed significance $(P<0.01)$. For patient-reported outcomes, the mean HOOS and SF-12 scores significantly improved at the final follow-up $(P<0.01)$ (Table 1$)$. Two of our patients were lost to follow-up.

Pre-operatively, the mean clinically measured LLD for unilateral THA was $4.2 \mathrm{~cm}$ (range, $2.1-6.5 \mathrm{~cm}$ ) and it significantly improved to $1.1 \mathrm{~cm}$ (range, $0.6-1.4 \mathrm{~cm}$ ) at the latest follow-up $(P<0.01)$, and no patients had $>2 \mathrm{~cm}$ LLD postoperatively. At the final follow-up time, limp was moderate in three hips (5.4\%), slight in eleven (19.6\%), and none in $42(75 \%)$ in the whole patient cohort. Moreover, positive Trendelenburg sign was presented in one patient (one hip) postoperatively. We did not include the subjects with opposite side $\mathrm{DDH}$ or other contralateral hip deformity.

The preoperative VAS score for low back pain was rated as severe in 10 patients (23.8\%), moderate in 28 patients $(66.7 \%)$, and mild in 4 patients $(9.5 \%)$. At the final follow-up, the low back pain was moderate in 3 patients $(7.1 \%)$, mild in 12 patients $(28.6 \%)$, and none in 27 patients $(64.3 \%)$. The low back pain improved over time, and the improvement was significant at every follow-up time point $(P<0.01)$ (Table 1 , Fig. 2$)$.

\section{Radiographic evaluation}

All acetabular components were placed in the position of true acetabulum (Fig. 3). Bone union of osteotomy site was archived in fifty-four hips with no complication, and the mean time to bone union was 6 months (range, 4-9 months). Serial radiographs showed two cases (4.1\%) of nonunion at osteotomy site. One patient was treated successfully with bone grafting and medication, and showed bone union of the osteotomy site within 8 months without any complication at last follow-up time; the other one showed progressive stem subsidence and then the signs of radiographic loosening through the postoperative period, and it was revised with fully porous-coated stem at postoperatively 3.4 years and the new femoral component was stable at last followup time. Femoral stem that remained in situ after THA on radiographs indicated bone ingrowth in fifty hips $(89.3 \%)$ at final follow-up time. Radiographic analyses of the femoral stem revealed radiolucency in three patients $(6.1 \%)$ (three hips). However, no signs of progressive subsidence or loosening of femoral component were observed at the last follow-up in any hip. One patient $(2.0 \%)$ (one hip) suffered from periprosthetic fracture around the femoral stem at 4.2 years, which was stabilized with a longer stem and cerclage wires, and fully healed without further complication. One patient $(2.0 \%)$ (one hip) sustained peri-prosthetic fracture of distal femur at 5 years, and cerclage wires and bone graft were applied successfully for fixation. In addition, two patients (4.1\%) (two hips) sustained acetabular fracture and loosening of the acetabular cup at postoperative five and seven years, respectively. They have been successfully treated by cup revision and bone grafting with no further complication (Table 2). Asymptomatic Brooker class-I and II heterotopic ossification were observed in three and two hips, respectively (Fig. 4). In addition, focal osteolysis was observed in five hips (Zone I in one hip, Zone II in two hips, and Zone 3 in two hips) on follow-up radiographs, but the cups showed bony incorporation and no surgery was required (Fig. 4). 


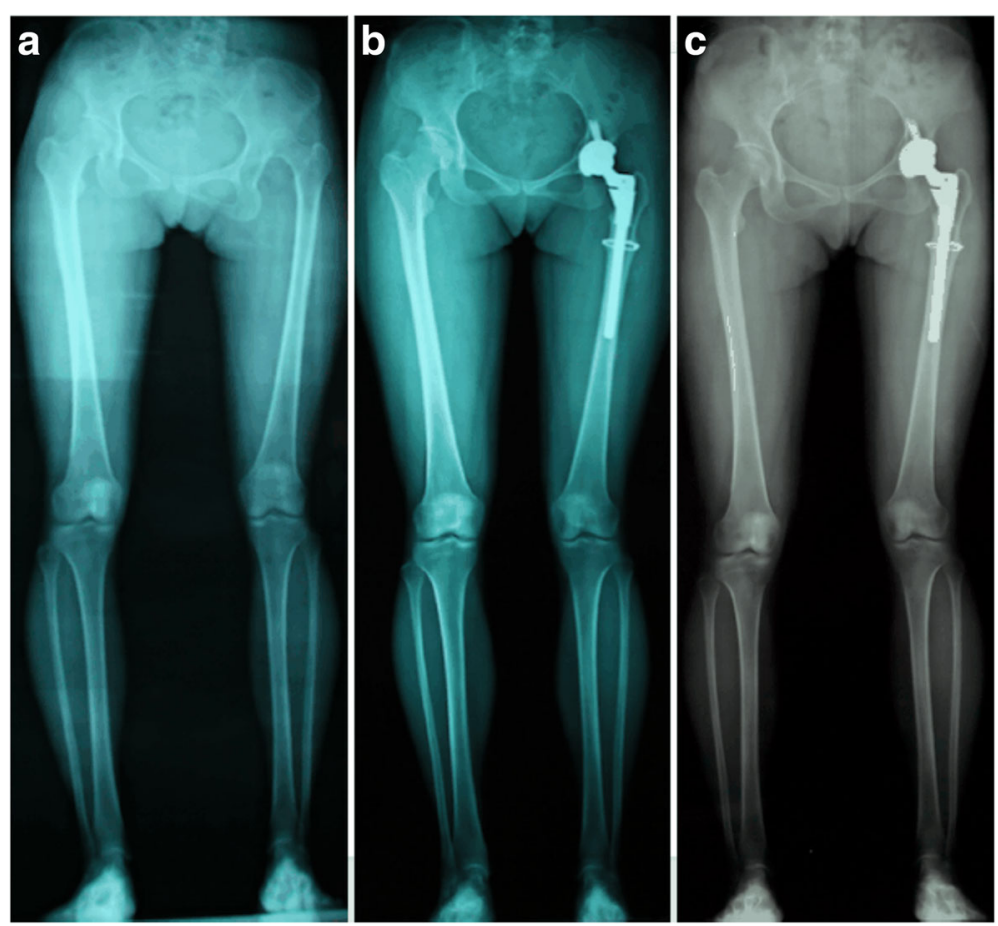

Fig. 2 Full-length radiographs of a twenty-five-year-old woman with unilateral Crowe type-IV DDH. The serial post-operative radiographic image showed that pelvic inclination was normalized over time. (a) Pre-operative radiographic image; (b) Radiographic image after four years follow-up; (c) Radiographic image after nine years follow-up

\section{Complications}

According to the Clavien-Dindo classification, the classification of surgical complications was grade I in 33 patients $(67.3 \%)$, grade II in 9 patients $(18.4 \%)$, and grade III in 7 patients (14.3\%), respectively [28]. At the beginning of the series, an intraoperative nondisplaced fracture occurred in proximal femur in two hips and in distal femur in two hips, which were successfully treated with internal fixation with cerclage bands without further complication. Three patients (6.1\%) (three hips) experienced transient nerve injury with weaknesses of ankle extension and dropfoot, who were treated with medication and physical therapy and fully recovered within 1 year without functional defects. Three patients (6.1\%) (three hips) had dislocation postoperatively. In two cases, dislocation was treated successfully with closed reduction without further sequelae (Fig. 4). In one case, dislocation recurred two

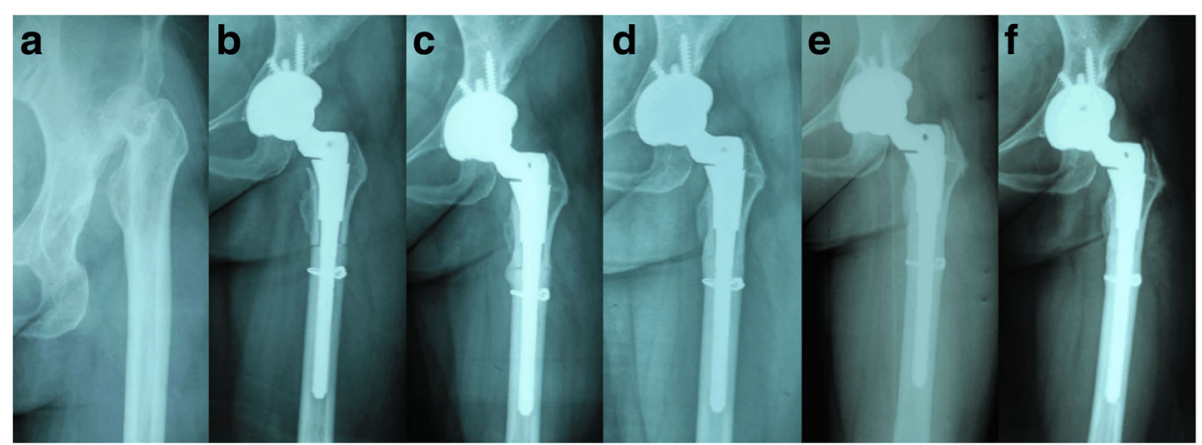

Fig. 3 Radiographs of a twenty-nine-year-old woman with unilateral Crowe type-IV DDH. (a) Preoperative anteroposterior view. (b) Post-operative radiographic image at four months follow-up. Total hip arthroplasty was combined with simultaneous transverse osteotomy. Cerclage wires were placed at osteotomy site, and the acetabulum was reconstructed with screws. (c) Post-operative radiographic image at one year follow-up. (d) At four years follow-up, bone union was detected at the osteotomy site without radiolucent lines around the stem. (e) At eight years follow-up, the femoral and acetabular components showed no radiographic signs of loosening. (f) Post-operative radiographic image at eleven years follow-up. The femoral and acetabular components were stable 
Table 2 Complications

\begin{tabular}{|c|c|c|c|}
\hline Case & Complication & Treatment & Results \\
\hline 5 & Dislocation at 7 days & Closed reduction & No recurred dislocation \\
\hline 13 & Dislocation at 2 years & Open reduction & No recurred dislocation \\
\hline 16 & Dislocation at 2 weeks & Closed reduction & No recurred dislocation \\
\hline 9 & Intraoperative femoral proximal fracture & Cerclage wires for fixation & No further complications \\
\hline 12 & Intraoperative femoral proximal fracture & Cerclage wires for fixation & No further complications \\
\hline 19 & Intraoperative femoral distal fracture & Cerclage wires for fixation & No further complications \\
\hline 24 & Intraoperative femoral distal fracture & Cerclage wires for fixation & No further complications \\
\hline 14 & Transient nerve injury & Medication and physical therapy & Full recovery \\
\hline 15 & Transient nerve injury & Medication and physical therapy & Full recovery \\
\hline 21 & Transient nerve injury & Medication and physical therapy & Full recovery \\
\hline 20 & $\begin{array}{l}\text { Nonunion of the osteotomy site, aseptic } \\
\text { loosening of femoral component }\end{array}$ & $\begin{array}{l}\text { Fully coated femoral component } \\
\text { used in revision, bone graft at } 3.4 \text { years }\end{array}$ & Bone ingrowth and stable \\
\hline 35 & Nonunion of the osteotomy site & Bone grafting and medication & Satisfactory clinical results \\
\hline 36 & Peri-prosthetic fracture around the femoral stem & $\begin{array}{l}\text { A longer stem and cerclage wires } \\
\text { used in revision at } 4.2 \text { years }\end{array}$ & Bone ingrowth and stable \\
\hline 40 & Peri-prosthetic fracture of distal femur & $\begin{array}{l}\text { Cerclage wires and bone graft } \\
\text { used in surgery at } 5 \text { years }\end{array}$ & Full healed \\
\hline 31 & $\begin{array}{l}\text { Acetabular fracture and thereby loosening of the } \\
\text { acetabular cup }\end{array}$ & $\begin{array}{l}\text { Cup revision and bone grafting } \\
\text { at } 5 \text { years }\end{array}$ & No further complication \\
\hline 17 & $\begin{array}{l}\text { Acetabular and greater trochanter fracture, and } \\
\text { screw breakage, and thereby loosening of the } \\
\text { acetabular cup }\end{array}$ & $\begin{array}{l}\text { Cup revision and bone grafting } \\
\text { at } 7 \text { years }\end{array}$ & No further complication \\
\hline
\end{tabular}

years after indexed THA and could not be resolved with closed reduction, and required open reduction. Moreover, four patients (8.2\%) complained of longer or shorter contralateral limb length. But no limbs were longer or shorter based on the result of radiographic measurement. No vascular complications, infection and deep venous thrombosis was recorded (Table 2). In summary, three $(6.1 \%)$ and four patients $(8.2 \%)$ required reoperation and revision surgery in the followup period, respectively.

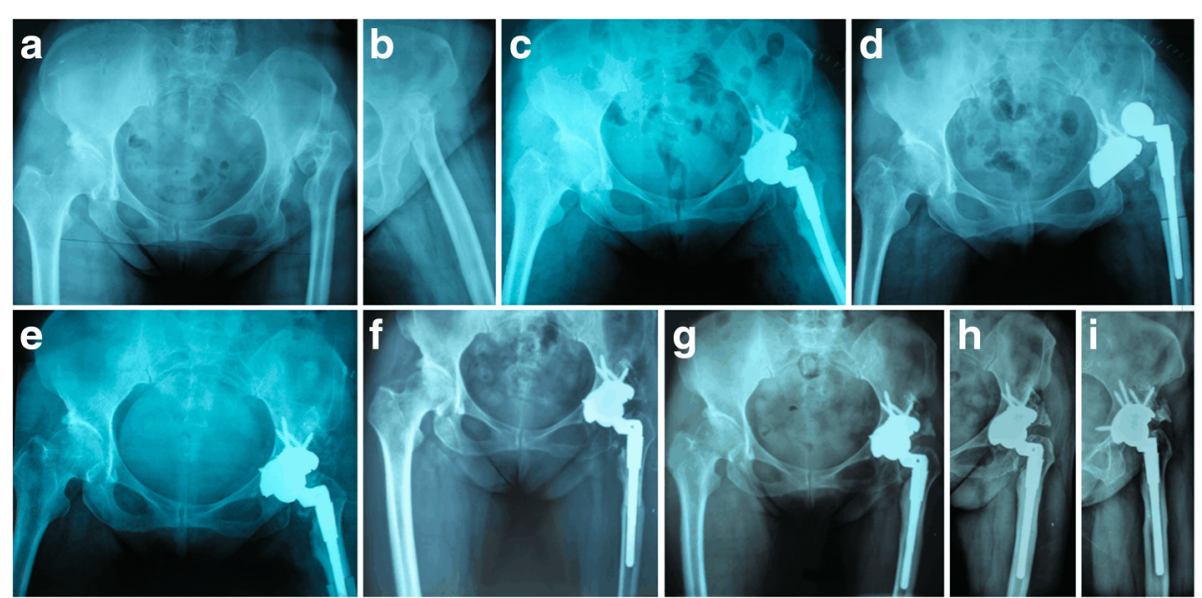

Fig. 4 Radiographs of a forty-two-year-old woman with unilateral Crowe type-IV DDH. (a-b) Pre-operative anteroposterior view. (c) Radiographic image at postoperative 1 day. Total hip arthroplasty was performed combined with transverse osteotomy in left hip. (d-e) Postoperative anteroposterior view showed dislocation at 1-week follow-up, which was treated successfully through closed relocation. (f) Radiographic image after five years follow-up showed that no radiolucent lines around the femoral and acetabular components were identified. But osteolysis around the stem at the distal tip and heterotopic ossification were identified. (g-i) At eleven years follow-up, the postoperative Harris hip score was 97, and the femoral stem was judged to be stable with bone ingrowth 


\section{Survivorship analysis}

Kaplan-Meier survivorship with an end point of any component revision was $92 \%$ (95\% confidence interval 84-99) at postoperative 10 years. Kaplan-Meier survivorship with an end point of aseptic loosening of the stem was 96\% (95\% confidence interval 87-99) at 10 years (Fig. 5).

\section{Discussion}

Crowe Type-III or IV developmental dysplasia is technically demanding for cementless THA in young patients, such as anatomical and biomechanical abnormalities, soft tissue contractures, and femoral deformities. In THA for severe DDH, satisfactory clinical and radiographic results have been reported with placement of the acetabular component in the true acetabulum and a femoral shortening osteotomy. However, the majority of studies reported series of a small number of patients who treated with THA combined with a subtrochanteric shortening osteotomy have included different surgical techniques with mixed groups of cemented and cementless implants or combinations of other osteotomy techniques, and the results are not consistent $[5,6,8,23$, 29-39] (Table 3). To our knowledge, this is the largest reported series of young patients with Crowe Type-III and IV DDH, in which the clinical and radiographic outcomes and related complications of exclusively THA combined with simultaneous transverse femoral subtrochanteric shortening osteotomy were evaluated. The major finding of this study was that the cementless THA combined with transverse subtrochanteric shortening osteotomy could improve function, resort a more normal limb, and provide stable fixation in hips with Crowe Type-III and IV DDH.

We noted some limitations of the current study. First, the number of patients was relatively small. Second, there was a retrospective study with no control group. Third, there was no evaluation of inter-observer reliability, and all operations were performed by five senior surgeons. Forth, low back pain was measured with VAS scale and lacks objective evidence in this study.

Previous studies reported that superior placement of the acetabular component could result in improper components orientation, unfavorable biomechanical environment and even higher rates of components loosening, especially in young patients with severe DDH $[40,41]$. Therefore, we placed all acetabular cups in true acetabulum and restored the anatomical center of hip rotation in our series. In addition, considering the young age of our series and the risk of the need for potential revision surgery, structural acetabular autograft was conducted in most cases. Linde et al. found that the rate of mechanical loosening of the acetabular cup was $13 \%$ in the group with the anatomical position of acetabular components compared with $42 \%$ in the group with superior placement of the acetabular cups at 15 years follow-up, and the difference revealed significance [40]. In our

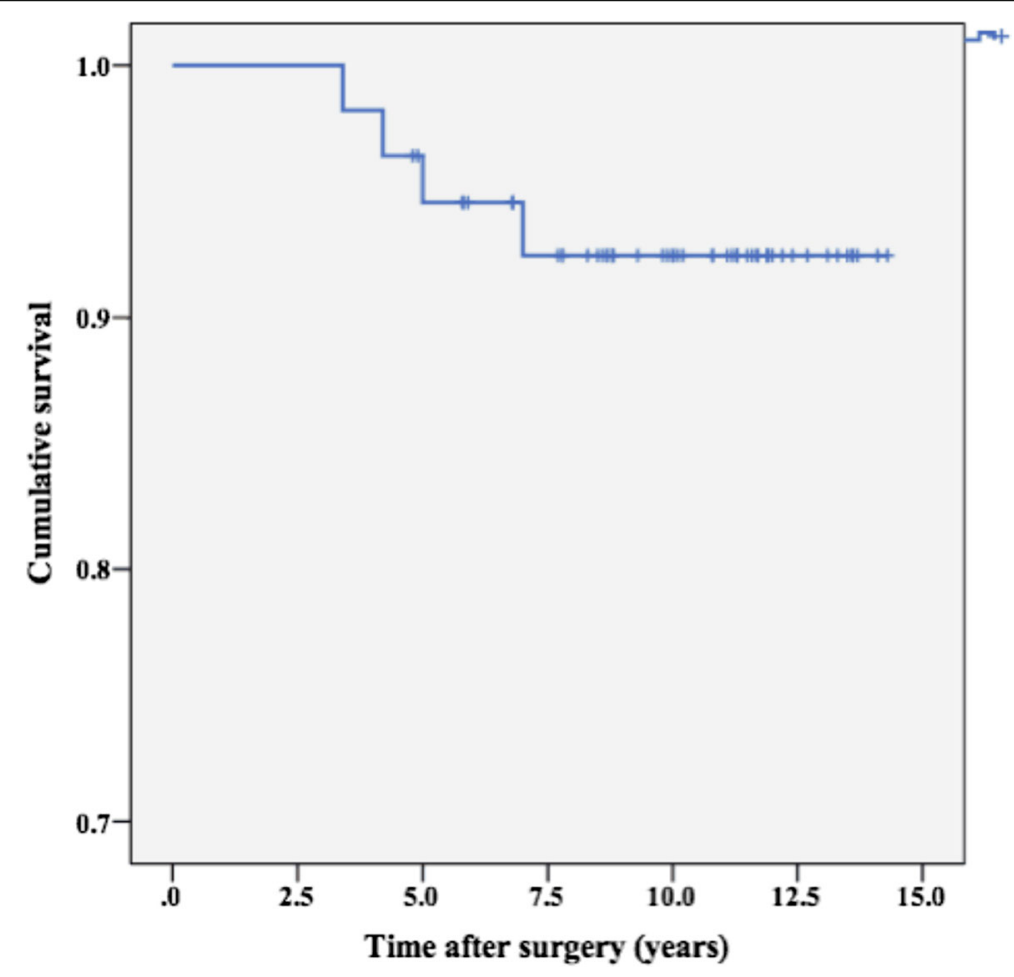

Fig. 5 Kaplan-Meier survivorship curve with revision for any component as the end points 


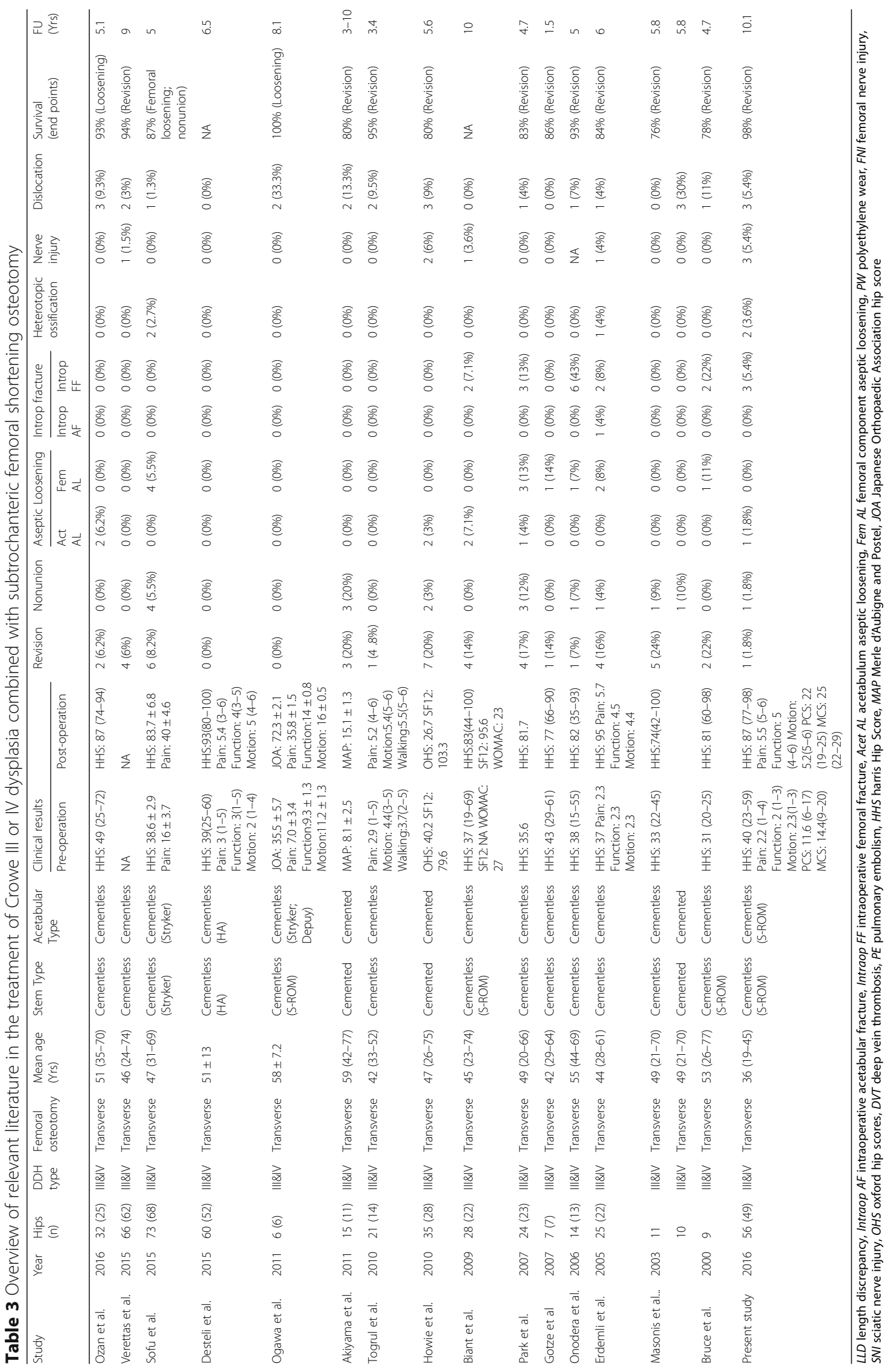


series, only two patients (two hips) showed acetabular component loosening (3.5\%) due to acetabular fracture and screw breakage.

Femoral shortening osteotomy is sometimes necessary to assist anatomic placement of acetabular cup without sciatic nerve compromise and excessive soft tissue tension [6,31]. Subtrochanteric osteotomies can be performed at different anatomic levels with various surgical techniques, including Z-shaped, transverse, oblique, and double Chevron $[2,10,11]$. A biomechanical study by Muratli et al. compared four different subtrochanteric osteotomies techniques, and demonstrated that there was no single inherent feature increasing the stability of the osteotomy designs [10]. Li et al. performed a metaanalysis comparing transverse and modified osteotomy regarding complications and survivorship and no difference was found [42]. And, transverse osteotomy may be recommended compared with other techniques due to technical simplicity, convenience of adjusting the anteversion angle, correction of the rotational deformity, preservation of the proximal femoral metaphysis, relatively short learning curve for precise performance and minimal damage of the periosteum in patients with severe DDH [5, 42]. Therefore, transverse subtrochanteric osteotomy was utilized in all hips. Common complications in femoral shortening osteotomy were intraoperative femoral fracture with rates from 5 to $24 \%[6,8,23]$ and nonunion at the osteotomy site with rates from 7 to $22 \%$ [31, 32, 35]. Great attention should be paid to narrow and straight femoral canals, which are so vulnerable to fracture during broaching or insertion of femoral stem. Our union rate of osteotomy site was $96.4 \%$. In our series, several methods were available to prevent nonunion. First, the interface between the osteotomy fragments should be cut as smoothly as possible. Second, autologous morselized bone grafts were used to augment the osteotomy gap, and the resected bone from femoral head was wrapped around osteotomy site. Third, great care was taken to minimize the damage of periosteum circumferentially during preparation of osteotomy sites and prophylactic cerclage wiring to keep the osteoblastic activity of periosteum. At the beginning of our series, no precautions were taken to decrease the risk of intraoperative femoral fracture, three cases of fracture were recorded that was successfully treated with cerclage bands and healed with no further complications. To obtain a strong fixation and minimize the risk of fracture, we subsequently paid more attention to fracture signs (e.g. sudden much more subsidence of the implant, rotational instability of stem at final insertion) during broaching and performed prophylactic cable fixation before the insertion of implant. The risk of intraoperative fracture decreased after prevention of fracture.
In this study, we consider dislocation as an important complication, which may compromise the press-fit between femoral component and femoral fragment and result in disengagement and early loosening of stem [43]. Total dislocation rates were $5.3 \%$ in our series that compared favorably with dislocation rates in other studies $[8,34,36,38]$. The relatively low incidence of dislocation can be interpreted by the stronger muscle strength in younger and more active patients, proper cup orientation, and restoration of abductor muscles lever arm and center of hip rotation [3, 6, 7, 31]. Another important complication was transient nerve injury in three patients, which were recovered with no weakness or numbness in the limb by the latest followup evaluation. Some researchers reported that femoral lengthening increase the incidence of nerve palsy, and thereby they recommended lengthening the femur by $<3 \mathrm{~cm},<4 \mathrm{~cm}$, or $<5 \mathrm{~cm}$ to avoid this complication [3, 12]. The transient nerve injuries occurred in our series due to relatively large amount of leg lengthening and less experience, and time-consumption at the beginning. We estimated the bone shortening based on preoperative templating, and believed that the leg lengthening of $<3.5 \mathrm{~cm}$ may decrease the risk of nerve injury [13].

The function outcomes, including HSS, HOOS, and SF-12, improved significantly in our study group comparable to the results reported in the literature, which may be attributed to the relief of preoperative pain, improvement of LLD, and restoration of abductor muscles lever arm and femoral offset $[5,34,35,38]$. In addition, we should pay more attention to chronic complications of the lumber spine and concomitant back pain, which was due to the compensation of lumber scoliosis and pelvic inclination for LLD. The results showed the significant improvement of patient's low back pain in this study, which can be explained by the two reasons: (1) LLD was decreased, and (2) thereby pelvic inclination and excess scoliosis or lordosis was normalized over time after this procedure.

It is generally accepted that the rates of implant failure were higher in young and more active patients due to a high grade of physical activity and functional demand, compared with elderly and inactive patients. As a result of high failure rate of components in cemented THA, cementless THA with ceramic on ceramic can provide long-term stable fixation and became the gold standard in younger cases. In addition, modular standard stem is very suitable in cases with severe DDH with a need for femoral osteotomy, which provides good fit and fill in dysplastic narrow femoral canal and stable fixation of the metaphyseal and diaphyseal fragments, and thereby obtains torsional stability [12,31]. With regards to the long-term survival, overall failure rate was $7.1 \%$ in our series, which was is comparable to other published studies recording a failure rate of $0-10 \%[31,44]$. 


\section{Conclusion}

For young patients with Crowe Type-III or IV DDH, THA is very difficult due to the abnormal anatomy of the proximal femur and acetabular structure. The midterm clinical and radiographic results of THA for these patients were good in our study. Thus, we demonstrated that cementless THA combined transverse subtrochanteric femoral shortening osteotomy in hips with Crowe Type-III or IV DDH is a reliable technique, which provides satisfactory outcomes, including acceptable rate of major lengthening-related complications, stable fixation of cementless implant, and significant improvement of function and relief of back pain.

\begin{abstract}
Abbreviations
DDH: developmental dysplasia of the hip; THA: Total hip arthroplasty; HOOS: Hip dysfunction and Osteoarthritis Outcome Score; HHS: Harris Hip Score; SF-12: 12-item short-form health survey questionnaire; MAP: Merle d'Aubigne and Postel; LLD: Limb-length discrepancy; VAS: Visual analogue scale
\end{abstract}

\section{Acknowledgements}

We would like to thank the relevant staff for guidance and assistance for their support and collaboration in our hospital.

\section{Funding}

None

\section{Availability of data and materials}

The datasets used and/or analyzed during the current study are available from the corresponding author on reasonable request.

\section{Authors' contributions}

ZKZ. and FXP. conceived and designed this study; DW, DHL, and QL collected the data; DW, HYW, YY and ZYL performed the statistical analysis; HYW, YY and ZYL prepared Tables 1-3; DW, HYW, YY and ZYL prepared Figs. 1-5; DW, QL and HYW wrote the manuscript; DW, DHL, and ZKZ revised this manuscript. All authors reviewed the final manuscript. All authors agree to be accountable for all aspects of the work.

\section{Ethics approval and consent to participate}

This study was approved by the local institutional review board of West China Hospital, Sichuan University (No. 201302008). Informed consent (including patients' details, images or videos) was obtained from all participants. All experiments were performed in accordance with relevant guidelines and regulations. This study was conducted in accordance to the Declaration of Helsinki.

\section{Consent for publication}

Not applicable.

\section{Competing interests}

The authors declare that they have no competing interests.

\section{Publisher's Note}

Springer Nature remains neutral with regard to jurisdictional claims in published maps and institutional affiliations.

\footnotetext{
Author details

${ }^{1}$ Department of Orthopedics, West China Hospital/West China School of Medicine, Sichuan University, 37\# Wuhou Guoxue road, Chengdu 610041, People's Republic of China. ${ }^{2}$ Key Laboratory of Birth Defects and Related Diseases of Women and Children, Ministry of Education, Sichuan University, Chengdu 610041, People's Republic of China. ${ }^{3}$ Department of Nursing, West China Second University Hospital/West China Women's and Children's Hospital, Chengdu 610041, China. ${ }^{4}$ State Key Laboratory of Oral Diseases, West China Hospital of Stomatology, Sichuan University, Chengdu 610041, People's Republic of China.
}

Received: 7 July 2017 Accepted: 17 November 2017

Published online: 25 November 2017

\section{References}

1. Noble PC, Kamaric E, Sugano N, Matsubara M, Harada Y, Ohzono K, Paravic V. Three-dimensional shape of the dysplastic femur: implications for THR. Clin Orthop Relat Res. 2003;417:27-40.

2. Bao N, Meng J, Zhou L, Guo T, Zeng X, Zhao J. Lesser trochanteric osteotomy in total hip arthroplasty for treating CROWE type IV developmental dysplasia of hip. Int Orthop. 2013;37(3):385-90.

3. Ahmed E. Ibrahim el G, Ayman B: Total hip arthroplasty with subtrochanteric osteotomy in neglected dysplastic hip. Int Orthop. 2015;39(1):27-33.

4. Eskelinen A, Helenius I, Remes V, Ylinen P, Tallroth K, Paavilainen T. Cementless total hip arthroplasty in patients with high congenital hip dislocation. J Bone Joint Surg Am. 2006;88(1):80-91.

5. Sofu H, Kockara N, Gursu S, Issin A, Oner A, Sahin V. Transverse subtrochanteric shortening osteotomy during Cementless Total hip arthroplasty in Crowe typeIII or IV developmental dysplasia. J Arthroplast. 2015;30(6):1019-23.

6. Howie CR, Ohly NE, Miller B. Cemented total hip arthroplasty with subtrochanteric osteotomy in dysplastic hips. Clin Orthop Relat Res. 2010;468(12):3240-7.

7. Zagra L, Bianchi L, Mondini A, Ceroni RG. Oblique femoral shortening osteotomy in total hip arthroplasty for high dislocation in patients with hip dysplasia. Int Orthop. 2015:39(9):1797-802.

8. Togrul E, Ozkan C, Kalaci A, Gulsen M. A new technique of subtrochanteric shortening in total hip replacement for Crowe type 3 to 4 dysplasia of the hip. J Arthroplast. 2010;25(3):465-70.

9. Mu W, Yang D, Xu B, Mamtimin A, Guo W, Cao L. Midterm outcome of Cementless Total hip arthroplasty in Crowe IV-Hartofilakidis type III developmental dysplasia of the hip. J Arthroplast. 2016;31(3):668-75.

10. Muratli KS, Karatosun V, Uzun B, Celik S. Subtrochanteric shortening in total hip arthroplasty: biomechanical comparison of four techniques. J Arthroplast. 2014;29(4):836-42.

11. Yalcin N, Kilicarslan K, Karatas F, Mutlu T, Yildirim H. Cementless total hip arthroplasty with subtrochanteric transverse shortening osteotomy for severely dysplastic or dislocated hips. Hip Int. 2010;20(1):87-93.

12. Takao M, Ohzono K, Nishii T, Miki H, Nakamura N, Sugano N. Cementless modular total hip arthroplasty with subtrochanteric shortening osteotomy for hips with developmental dysplasia. J Bone Joint Surg Am. 2011;93(6): 548-55.

13. Wang D, Li LL, Wang HY, Pei FX, Zhou ZK. Long-term results of Cementless Total hip arthroplasty with subtrochanteric shortening osteotomy in Crowe type IV developmental dysplasia. J Arthroplast. 2016:32(4):1211-19.

14. Krych AJ, Howard JL, Trousdale RT, Cabanela ME, Berry DJ. Total hip arthroplasty with shortening subtrochanteric osteotomy in Crowe type-IV developmental dysplasia. J Bone Joint Surg Am. 2009;91(9):2213-21.

15. Charity JA, Tsiridis E, Sheeraz A, Howell JR, Hubble MJ, Timperley AJ, Gie GA. Treatment of Crowe IV high hip dysplasia with total hip replacement using the Exeter stem and shortening derotational subtrochanteric osteotomy. J Bone Joint Surg Br. 2011;93(1):34-8.

16. Imam MA, Fathalla I, Holton J, Nabil M, Kashif F. Cementless Total hip replacement for the Management of Severe Developmental Dysplasia of the hip in the middle eastern population: a prospective analysis. Front Surg. 2016;3:31.

17. Hasegawa Y, Iwase T, Kanoh T, Seki T, Matsuoka A. Total hip arthroplasty for Crowe type developmental dysplasia. J Arthroplast. 2012;27(9):1629-35.

18. Parvizi J, Pour AE, Hillibrand A, Goldberg G, Sharkey PF, Rothman RH. Back pain and total hip arthroplasty: a prospective natural history study. Clin Orthop Relat Res. 2010;468(5):1325-30.

19. Swarup I, Marshall AC, Lee YY, Figgie MP. Implant survival and patientreported outcomes after total hip arthroplasty in young patients with developmental dysplasia of the hip. Hip Int. 2016;26(4):367-73.

20. Zeng WN, Wang FY, Chen C, Zhang Y, Gong XY, Zhou K, Chen Z, Wang D, Zhou ZK, Yang L. Investigation of association between hip morphology and prevalence of osteoarthritis. Sci Rep. 2016;6:23477.

21. Zeng WN, Zhou K, Zhou ZK, Shen B, Yang J, Kang PD, Pei FX. Comparison between drainage and non-drainage after total hip arthroplasty in Chinese subjects. Orthop Surg. 2014;6(1):28-32.

22. Li W, Zhang W, Bai G, Huang Z, Shen R. Total hip arthroplasty for treatment of Crowe type IV congenital dysplasia of hip with dislocation in adults. Zhongguo Xiu Fu Chong Jian Wai Ke Za Zhi. 2013;27(10):1153-6. 
23. Masonis JL, Patel JV, Miu A, Bourne RB, McCalden R, Macdonald SJ, Rorabeck $\mathrm{CH}$. Subtrochanteric shortening and derotational osteotomy in primary total hip arthroplasty for patients with severe hip dysplasia: 5-year follow-up. J Arthroplast. 2003;18(3 Suppl 1):68-73.

24. DeLee JG, Charnley J. Radiological demarcation of cemented sockets in total hip replacement. Clin Orthop Relat Res. 1976;121:20-32.

25. Brooker AF, Bowerman JW, Robinson RA, Riley LH, Jr.: Ectopic ossification following total hip replacement. Incidence and a method of classification. J Bone Joint Surg Am 1973, 55(8):1629-1632.

26. Gruen TA, McNeice GM, Amstutz HC. "modes of failure" of cemented stemtype femoral components: a radiographic analysis of loosening. Clin Orthop Relat Res. 1979;141:17-27.

27. Engh CA, Massin P, Suthers KE. Roentgenographic assessment of the biologic fixation of porous-surfaced femoral components. Clin Orthop Relat Res. 1990; 257:107-28.

28. Dindo D. The Clavien-Dindo classification of surgical complications. Springer London. 2014;250:187-96.

29. Desteli EE, Imren Y, Tan E, Erdogan M, Ozcan H. Clinical results of cementless total hip arthroplasty with shortening osteotomy for high dislocation with developmental dysplasia. Acta Orthop Belg. 2015;81(1):30-5.

30. Verettas DA, Chloropoulou P, Xarchas K, Drosos G, Ververidis A, Kazakos K. Small diameter acetabulum and femoral head in total hip arthroplasty for developmental dysplasia of the hip, with no femoral osteotomy. Hip Int. 2015;25(3):209-14.

31. Biant LC, Bruce WJ, Assini JB, Walker PM, Walsh WR. Primary total hip arthroplasty in severe developmental dysplasia of the hip. Ten-year results using a cementless modular stem. J Arthroplast. 2009;24(1):27-32.

32. Bruce WJ, Rizkallah SM, Kwon YM, Goldberg JA, Walsh WR. A new technique of subtrochanteric shortening in total hip arthroplasty: surgical technique and results of 9 cases. J Arthroplast. 2000;15(5):617-26.

33. Erdemli B, Yilmaz C, Atalar H, Guzel B, Cetin I. Total hip arthroplasty in developmental high dislocation of the hip. J Arthroplast. 2005;20(8):1021-8.

34. Onodera S, Majima T, Ito H, Matsuno T, Kishimoto T, Minami A. Cementless total hip arthroplasty using the modular S-ROM prosthesis combined with corrective proximal femoral osteotomy. J Arthroplast. 2006;21(5):664-9.

35. Park MS, Kim KH, Jeong WC. Transverse subtrochanteric shortening osteotomy in primary total hip arthroplasty for patients with severe hip developmental dysplasia. J Arthroplast. 2007;22(7):1031-6.

36. Ozan F, Uzun E, Gurbuz K, Koyuncu S, Altay T, Kayali C. Total hip arthroplasty in the developmental dysplasia of the hip using transverse subtrochanteric osteotomy. J Orthop. 2016;13(4):259-63.

37. Akiyama H, Kawanabe K, Yamamoto K, Kuroda Y. So K, Goto K, Nakamura T: cemented total hip arthroplasty with subtrochanteric femoral shortening transverse osteotomy for severely dislocated hips: outcome with a 3- to 10year follow-up period. J Orthop Sci. 2011;16(3):270-7.

38. Ogawa H, Ito Y, Shinozaki M, Matsumoto K, Shimizu K. Subtrochanteric transverse shortening osteotomy in cementless total hip arthroplasty achieved using a modular stem. Orthopedics. 2011;34(3):170.

39. Gotze C, Winkelmann W, Gosheger G, Rodl R. Is there a need of an additional extramedullary fixation in transverse subtrochanteric shortening in primary total hip arthroplasty for patients with severe hip dysplasia? Short-term experience in seven patients with congenital dislocation. Z Orthop Unfall. 2007; 145(5):568-73.

40. Linde F, Jensen J, Pilgaard S. Charnley arthroplasty in osteoarthritis secondary to congenital dislocation or subluxation of the hip. Clin Orthop Relat Res. 1988; 227:164-71.

41. Makita H, Inaba Y, Hirakawa K, Saito T. Results on total hip arthroplasties with femoral shortening for Crowe's group IV dislocated hips. J Arthroplast. 2007;22(1):32-8

42. Li C, Zhang C, Zhang M, Ding Y. Comparison of transverse and modified subtrochanteric femoral shortening osteotomy in total hip arthroplasty for developmental dysplasia of hip: a meta-analysis. BMC Musculoskelet Disord. 2014;15:331.

43. Zhu J, Shen C, Chen X, Cui Y, Peng J, Cai G. Total hip arthroplasty with a non-modular conical stem and transverse subtrochanteric osteotomy in treatment of high dislocated hips. J Arthroplast. 2015;30(4):611-4.

44. Vermersch T, Viste A, Desmarchelier R, Fessy MH. Prospective longitudinal study of one hundred patients with total hip arthroplasty using a secondgeneration cementless dual-mobility cup. Int Orthop. 2015;39(11):2097-101.

\section{Submit your next manuscript to BioMed Central and we will help you at every step:}

- We accept pre-submission inquiries

- Our selector tool helps you to find the most relevant journal

- We provide round the clock customer support

- Convenient online submission

- Thorough peer review

- Inclusion in PubMed and all major indexing services

- Maximum visibility for your research

Submit your manuscript at www.biomedcentral.com/submit
Ciomed Central 